\title{
Réintroduire le patrimoine de l'eau parmi les communs
}

Pour un développement alternatif et durable au Maroc

Re-establishing water heritage among the commons. For an alternative and sustainable development in Morocco

Sandrine Simon

\section{OpenEdition}

Journals

Édition électronique

URL : https://journals.openedition.org/insituarss/599

DOI : 10.4000/insituarss.599

ISSN : 2680-4972

\section{Éditeur}

Ministère de la Culture

Référence électronique

Sandrine Simon, «Réintroduire le patrimoine de l'eau parmi les communs », In Situ. Au regard des sciences sociales [En ligne], 2 | 2021, mis en ligne le 18 mars 2021, consulté le 23 janvier 2023. URL http://journals.openedition.org/insituarss/599 ; DOI : https://doi.org/10.4000/insituarss.599

Ce document a été généré automatiquement le 23 janvier 2023.

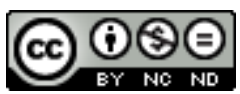

Creative Commons - Attribution - Pas d'Utilisation Commerciale - Pas de Modification 4.0 International - CC BY-NC-ND 4.0

https://creativecommons.org/licenses/by-nc-nd/4.0/ 


\title{
Réintroduire le patrimoine de l'eau parmi les communs
}

\author{
Pour un développement alternatif et durable au Maroc \\ Re-establishing water heritage among the commons. For an alternative and \\ sustainable development in Morocco
}

\section{Sandrine Simon}

\section{Crise de l'eau : le besoin d'une double approche par les communs et par le patrimoine}

1 L'eau est un bien environnemental commun par excellence. Indispensable à la survie non seulement des humains mais aussi des systèmes écologiques, il répond aux besoins les plus fondamentaux et doit être partagé entre des communautés qui, bien qu'habitant toutes une même planète bleue, n'ont pas le même accès à cette ressource. Pour cette raison, de nombreux auteurs ont affirmé que les guerres du xxI siècle naîtraient de conflits centrés sur le problème de l'eau (Petitjean 2016 ; Lasserre 2009).

Cet article se concentre sur un pays semi-aride, le Maroc, qui, face à une montée rapide du stress hydrique, se doit de choisir le meilleur mode de gestion de ce bien commun le plus essentiel aux hommes et à l'économie : l'eau. Cependant, ce choix n'est pas tâche aisée. Il impose notamment de tenir compte de l'approche islamique de l'eau qui interdit la vente de ce «don de Dieu » vital et est, de ce fait, en opposition avec la politique de marchandisation de l'eau pourtant désormais très répandue. Dans ce contexte, le patrimoine de l'eau (qui regroupe les savoir-faire traditionnels, le droit coutumier, ainsi que les rites et usages traditionnels de l'eau) pourrait aider à apporter des solutions plus durables que celles qui furent adoptées au nom de la modernité aux lendemains de l'Indépendance. En effet, la politique de "régionalisation avancée ${ }^{1}$ ", l'appel à de nouveaux modes de gouvernance, la nécessité de faire face à la marginalisation et à l'appauvrissement du monde rural, et les débats récents sur le 
choix d'un autre mode de développement économique sont autant de raisons pour penser différemment la relation à l'environnement.

Pour ce faire, après avoir situé les ressources hydriques et les savoir-faire dans le contexte des biens communs et du patrimoine, nous proposerons trois types de dialogue entre ce que recouvrent ces deux notions. Le premier souligne la dimension conflictuelle introduite par la modernité qui a « dé-communisé » (isolé et dévalorisé) le patrimoine de l'eau et conduit à des appropriations exclusives d'une ressource qui devrait pourtant être considérée comme commune. Le second démontre un retour actuel à une forte sensibilisation de la société civile à l'importance de l'eau comme bien commun mais aussi comme patrimoine, dans un contexte d'appartenance culturelle et écologique. Enfin le troisième explore les formes possibles d'identification des modes de développement alternatif à travers une nouvelle politique de l'eau qui défend la protection des communs par le biais de la protection du patrimoine.

\section{Biens communs vs patrimoine : quelle approche pour mieux gérer l'eau?}

Besoin de base ou marchandise, formule chimique $\left(\mathrm{H}_{2} \mathrm{O}\right)$ ou dimension spirituelle, l'eau peut être décrite de différentes façons, toutes aussi importantes les unes que les autres et toutes reliées. Nous nous proposons tout d'abord d'explorer comment les notions de commun et de patrimoine ont pu émerger dans le contexte des stratégies de gestion de l'eau au Maroc et ailleurs.

5 Pour ce faire, le passage par l'approche économique est incontournable car c'est cette approche qui tend à prévaloir depuis des décennies dans l'introduction de la "valeur " dans nos sociétés. S'agissant de l'eau par exemple, pour les économistes, la pénurie liée aux changements climatiques ${ }^{2}$ appelle toutes sortes de mesures complémentaires pour parvenir à une meilleure gestion de cette ressource, à commencer par son assimilation à un nouveau type de capital naturel, auquel on accorde (enfin!) une valeur. Jusqu'à présent pourtant, les économistes avaient considéré l'eau comme une ressource disponible en quantité illimitée et s'en étaient donc désintéressés. En pratique, cette valeur économique s'est traduite par l'introduction de droits de propriété des ressources hydriques, dans une logique marchande. Cependant, comme l'expliquent Olivier Petit et Bruno Romagny (2009: 33), «le recours exclusif à ce système d'allocation sous-estime le caractère collectif de la gestion de ces ressources, néglige en grande partie les questions d'équité au profit du critère d'efficience et éprouve de sérieuses difficultés pour appréhender le long terme ». 
Figure 1

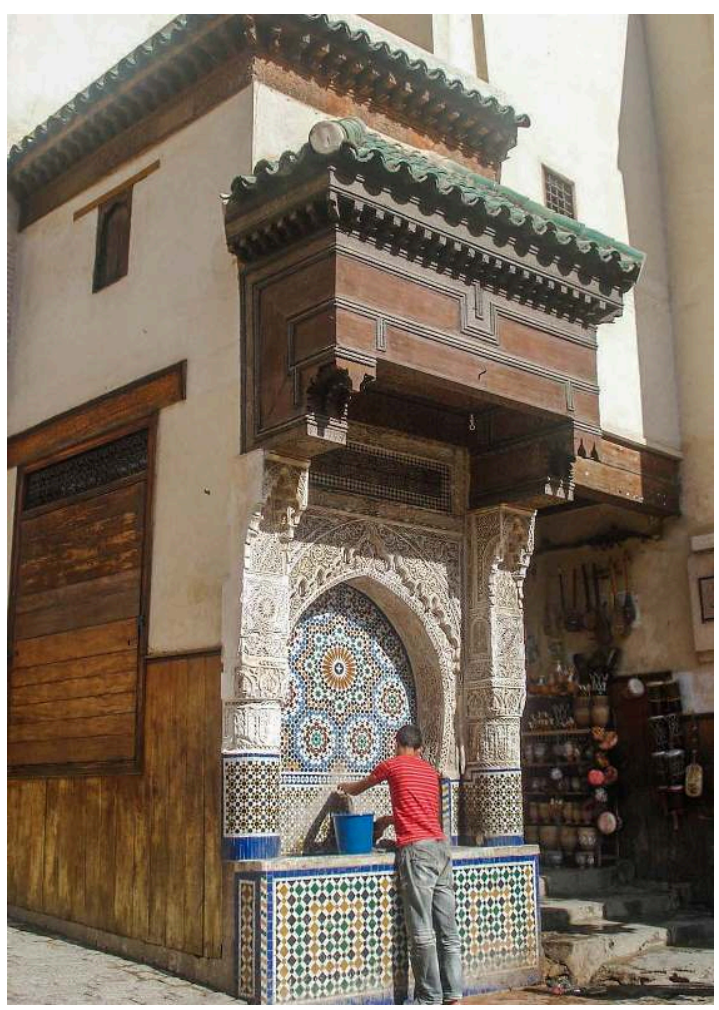

Fontaine Nejjarine dans la médina de Fès. L'eau était (elle l'est toujours dans certains endroits des vieilles médinas marocaines) traditionnellement accessible gratuitement au public, grâce aux fontaines murales de la vieille ville. Cet accès gratuit à la ressource est progressivement remplacé par un paiement qui inclut les services concernant le traitement des eaux usées.

(c) Photo Sandrine Simon.

Dans le contexte du xxi siècle, l'ouverture à de nouvelles approches s'impose. En particulier, le courant de pensée sur les ressources communes (Ostrom 1990) et l'approche patrimoniale, en nous orientant vers une perspective globale intégrant des critères d'équité dans l'allocation, la gestion et la transmission des ressources, pourraient proposer des cadres analytiques de développement particulièrement prometteurs. Leur objectif est de « dépasser l'interprétation pessimiste de la tragédie des communs (Hardin 1968) ${ }^{3}$ pour fonder une analyse plus pragmatique des mécanismes d'action collective et de gouvernance des ressources communes, appliquée principalement dans les pays en développement » (Wade 1988; Ostrom 1990 ; Bromley 1992 ; tous trois cités dans Petit \& Romagny 2009 : 33).

7 Le courant des ressources communes, né dans les années 1980 dans le monde anglosaxon, les décrit globalement comme des ressources naturelles, culturelles ou artificielles partagées par différents utilisateurs, dont l'exploitation peut donner lieu à une rivalité, souvent à l'origine de leur dégradation. Un des exemples de ces « common pool resources » est l'eau, dont l'usage est caractérisé par une grande concurrence, surtout en situation d'aridité. La liste des biens communs s'est aujourd'hui étendue (et aussi complexifiée) allant des biens matériels aux biens immatériels et concerne aujourd'hui quasiment tous les registres de la vie sociale et environnementale, à différentes échelles (Michon 2019 : 9). Ce courant de pensée a donné lieu à une analyse approfondie des régimes de propriété ou des modes d'appropriation qui dépasse la dichotomie simpliste entre propriété privée et propriété étatique. En effet, comme 
l'explique Perrine Michon (2019: 49) : «Une des leçons des analyses d'Ostrom est que ce ne sont pas les qualités intrinsèques des ressources qui font les communs mais la communauté et les règles qui la dirigent. Sont communes les ressources qu'une communauté décide de rendre telles en se donnant des règles pour en gouverner l'usage. [...] La propriété ne renvoie pas seulement au rapport - d'appropriation - entre la personne et une chose, c'est plutôt un rapport entre les personnes à propos de certaines choses sur lesquelles il y a une entente préalable. »

Figure 2

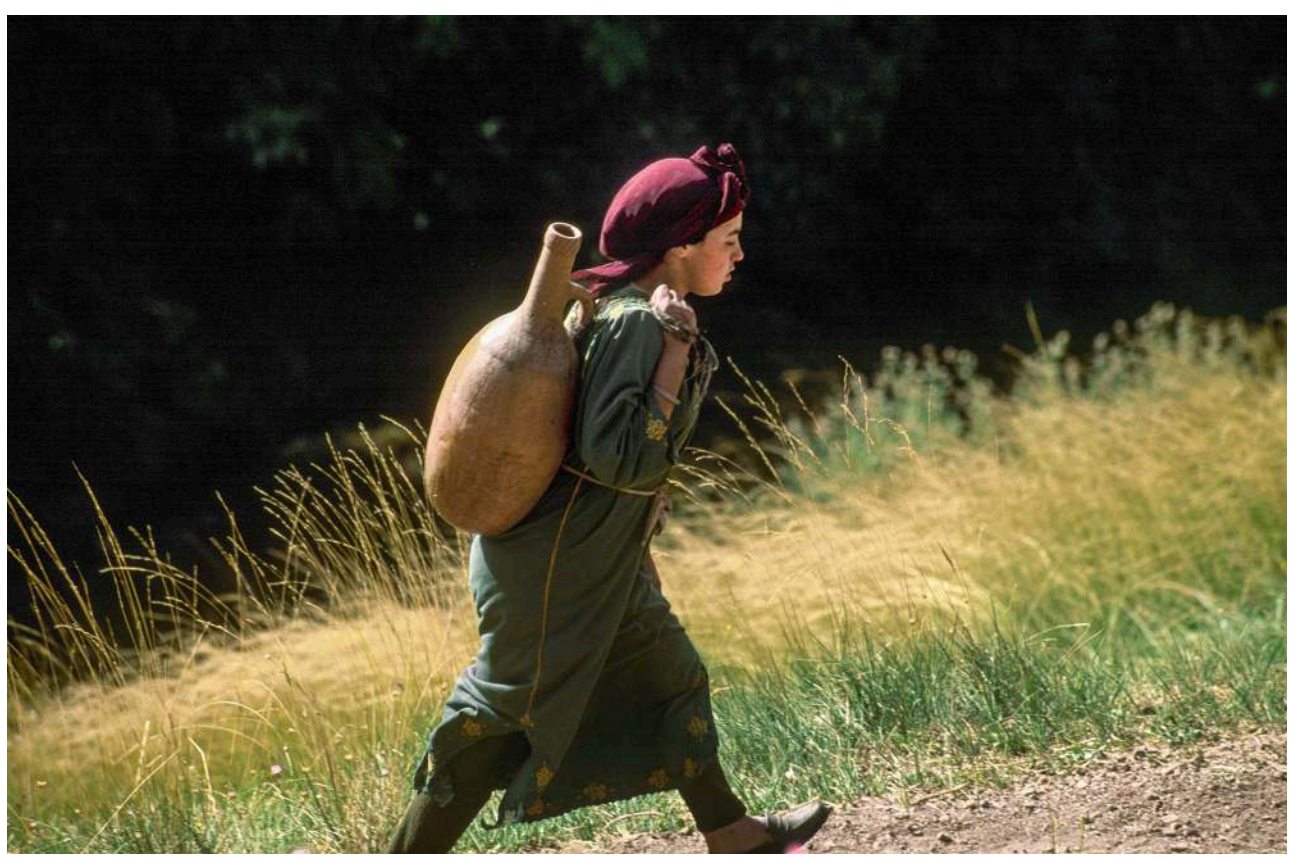

Jeune fille de corvée d'eau dans le Haut Atlas marocain, 2009. L'accès à l'eau dans les régions rurales du Maroc reste difficile.

(c) Photo Olivier Barrière, distr. IRD (http://www.indigo.ird.fr).

De ce fait, on comprend que les dimensions sociologiques et culturelles soient particulièrement importantes pour mieux comprendre et gérer les biens communs. Dans cette optique, on peut rappeler la définition des biens culturels communs, considérés comme "systèmes de ressources intellectuelles, disponibles dans un espace géographique ou virtuel »(Fiorentino, Friel, Marelli \& Santagata 2010), dont la spécificité est «d'être non rivaux et non exclusifs et dont l'utilisation n'épuise pas la ressource. L'usage des biens culturels communs peut inspirer et encourager de nouvelles créations et de nouveaux processus créatifs " (Cominelli 2012: 88). Les savoir-faire traditionnels relatifs à la gestion de l'eau au Maroc sont un de ces biens culturels qui nous intéressent ici. Comme l'explique Francesca Cominelli (2012: 89) "d'un point de vue holistique, les savoir-faire peuvent entrer dans la définition des biens culturels communs. Comme ces derniers, les savoir-faire sont localisés dans le temps et dans l'espace, ancrés dans un territoire précis en raison de la disponibilité de ressources spécifiques, naturelles, patrimoniales et infrastructurelles et d'un capital humain et social fort. Le système d'éléments composant un savoir-faire est en mesure d'évoluer, de se transmettre de père en fils, d'une famille à l'autre, au sein d'une communauté. Ce savoir-faire appartient à la communauté, il est incorporé et détenu 
par ses membres, il n'est pas immuable mais continue à innover, tout en se transmettant $»$.

On ne peut s'empêcher de voir ici un clin d'œil d'ordre sémantique au terme " patrimoine commun » associé aux ressources naturelles et en particulier à l'eau dès les années 1960, ainsi qu'au terme « patrimoine culturel immatériel » (PCI), défini en 2003 par la Convention pour la sauvegarde du PCI comme la source principale de la diversité culturelle ainsi que de la créativité comprenant «les pratiques, représentations, expressions, connaissances et savoir-faire - ainsi que les instruments, objets, artefacts et espaces culturels qui leur sont associés » (Unesco 2003 : 3). Le rôle des communautés dans la reconnaissance, le maintien, la transmission de ce patrimoine culturel immatériel est absolument central (Conseil de l'Europe 2005).

Figure 3

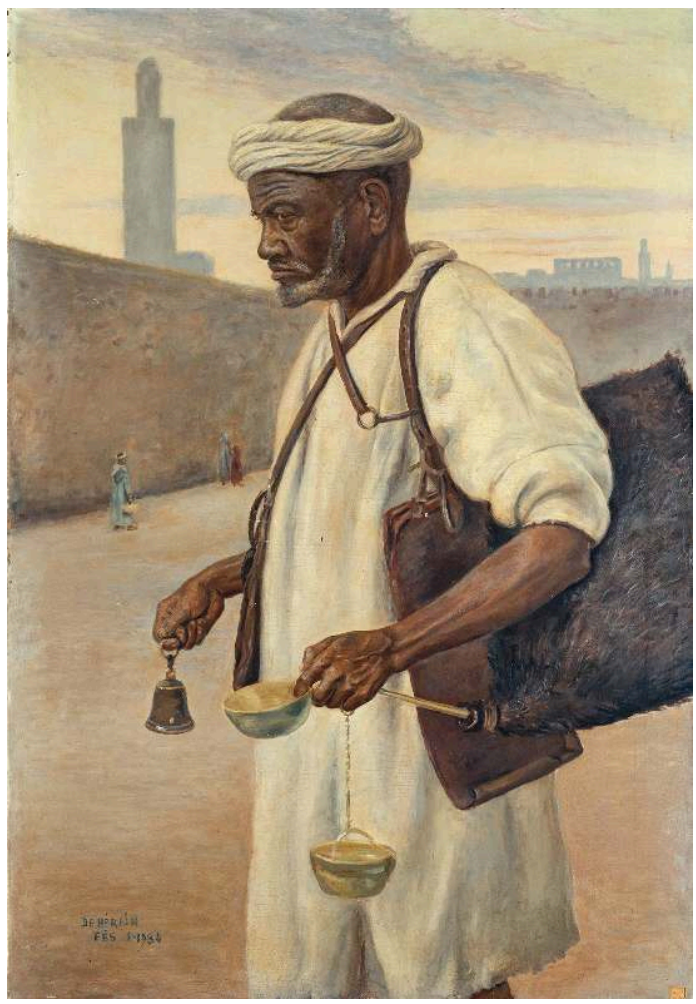

Porteur d'eau de Fès, François de Hérain (1877-1962), huile sur toile conservée au musée du quai Branly - Jacques-Chirac. Aujourd'hui comme hier, les porteurs d'eau distribuent de l'eau en contrepartie de quelques pièces, selon la volonté du client ou selon ce que ce dernier peut se permettre de payer.

Reproduction Daniel Arnaudet (RMN-Grand Palais).

Ainsi, le patrimoine n'existe pas en soi, mais en tant qu'il est identifié comme tel par ses détenteurs. Cette façon de penser met en lumière les dimensions territoriales et temporelles de la gestion de l'eau, et appelle à la constitution de communautés permettant de l'envisager dans une perspective de développement durable (Petit 2015). Le patrimoine inclut à la fois ce que les hommes font, ce sur quoi ils travaillent, les raisons pour lesquelles ils le font, les émotions et croyances qui les motivent à le faire et donc leur relation vivante au milieu qui les entoure. Ce respect fondamental de l'acteur en tant qu'il est juge de ce qui compte permet donc aussi de développer des techniques de mise au jour de différents points de vue et de résolution des conflits, 
visant à associer l'ensemble des acteurs intéressés par la gestion des ressources, et conçues comme des techniques de copilotage permanent et évolutif des relations entre sociétés humaines et nature (Passet 1990).

Ce rapport de l'homme à la nature révèle donc que peuvent coexister différents types de patrimoine de l'eau, alors même que cette précieuse ressource, indispensable à la vie, est justement commune et unique. Selon Elinor Ostrom, les communautés ont un grand rôle à jouer dans sa préservation, mais les modes de préservation peuvent varier. Cette approche patrimoniale de la gestion de l'eau peut renvoyer à des objectifs tels que, par exemple, «éviter la surexploitation, conserver la ressource et contourner les conflits» (Maillefert 2008: 282-283). Elle s'inscrit donc dans l'espace (gestion en commun d'un bassin par exemple), dans le temps (transmission du droit coutumier, notamment) et identitaire (qualification de la ressource par des communautés du désert, ou de la montagne).

L'approche par le patrimoine immatériel, tout comme l'approche par les ressources communes développée par Ostrom, ont permis d'étendre le champ d'étude et la compréhension de notions telles que le patrimoine de l'eau et d'explorer davantage l'importance des communautés. De même, l'ouverture à ces concepts et la naissance de notions telles que le patrimoine mondial, grâce aux actions notamment de l'Unesco (Gravari Barbas \& Guichard $2003:$ 11), aident à comprendre à quel point la notion de patrimoine a été eurocentrée, et à envisager d'autres modes de protection des ressources et des communautés humaines. Les promesses de l'approche patrimoniale sont liées au fait qu'elle « vise la restauration de la qualité des milieux naturels avec le double souci de leur transmission et du maintien de l'identité des acteurs au sein du milieu dans lequel ils vivent» (Petit \& Romagny 2009: 30), encourageant donc des approches économiques de la gestion de nos ressources naturelles proches de l'écodéveloppement (Lazarev 2000) et du développement durable de plus en plus éloignés des principes de l'économie néoclassique jugés inappropriés. 


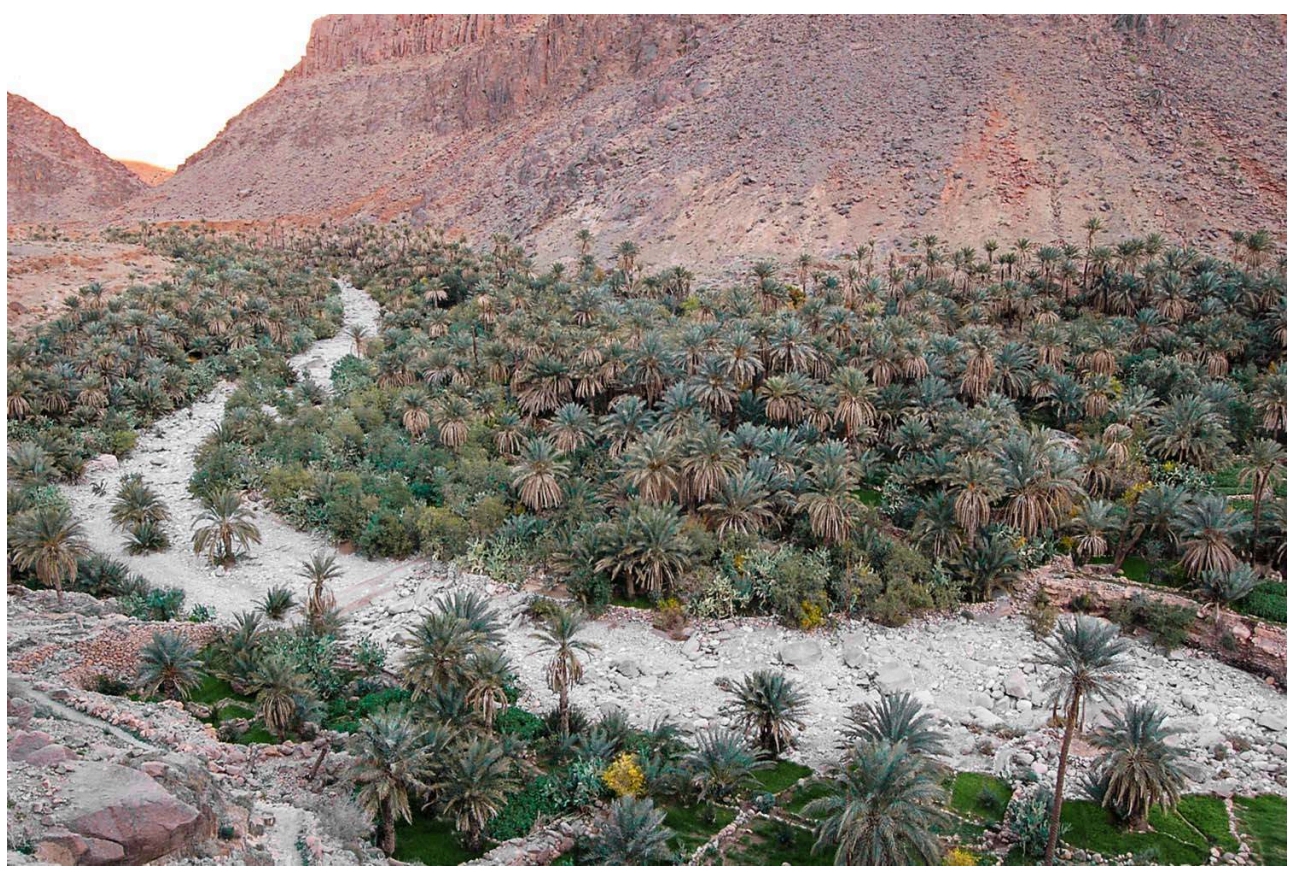

Les connaissances en matière de gestion de l'eau sont remarquables, surtout dans des régions arides où les hommes ont su apprendre à verdir le désert. Vallée d'Agouliz, sud marocain.

(c) Photo Sandrine Simon.

Ce sont précisément ces types d'approches alternatives de ce que signifie une gestion durable de l'eau qui nous intéressent dans le cadre du Maroc. Si la «Gestion intégrée des ressources en eau " (Gire), désormais encouragée dans ce pays, « est organisée à une échelle territoriale pertinente (bassin versant, fleuve, lac, aquifère), elle reprend un certain nombre d'idées sur la dimension patrimoniale de l'eau », tout en mettant "cependant encore en avant la nécessité d'une régulation marchande de l'eau, soulignant l'ambivalence de cette ressource à la fois patrimoine commun et bien marchand» (Petit \& Romagny 2009: 33). Est-il toutefois possible en ce début de $\mathrm{xxI}^{\mathrm{e}}$ siècle, de puiser dans les approches par les communs et le patrimoine pour faire face aux contraintes hydriques actuelles et répondre aux besoins des communautés humaines de façon plus appropriée et durable que par les approches modernes des lendemains de l'indépendance? Comme Petit et Romagny, nous sommes conduits à « interroger les couples individu-communauté, gestionnaire-usager, public-privé, court terme-long terme, local-global [...] comme des marqueurs du patrimoine commun » afin de "renouveler la réflexion sur ce que la théorie économique traditionnelle continue souvent à prendre pour un élément simplement assimilable au capital, alors même que de nouveaux cadres analytiques s'avèrent plus que jamais nécessaires » (ibid. : 32). 


\section{Trois modèles de dialogue entre bien commun et patrimoine : gestion traditionnelle vs Grande Hydraulique}

14 Le Maroc doit aujourd'hui faire face à de nombreux défis relatifs à l'eau. Aux besoins de son secteur agricole, consommateur de $88 \%$ de l'eau utilisée dans le pays, s'ajoutent ceux des villes rapidement grandissantes, en partie à cause d'un exode rural exacerbé, et le retard pris en matière de traitement des eaux usées en milieu urbain. Récemment, peu de temps après que le Printemps arabe a fleuri dans d'autres pays du Maghreb, de nombreuses communautés ont manifesté contre l'accroissement du prix de l'eau et les méfaits de technologies modernes telles que les forages qui encouragent la surexploitation des ressources hydriques souterraines. Parallèlement à cela, l'appauvrissement des milieux ruraux, dû en grande partie à une politique agricole tournée vers l'exportation et la mise en valeur de certaines régions privilégiées à l'époque du protectorat et après l'indépendance, ainsi que la construction de grands barrages et systèmes de transferts d'eau ont créé des déséquilibres écologiques et sociaux. Corriger ceux-ci nécessite de nouvelles approches.

Le développement durable, que le Maroc a inclus dans sa «Stratégie nationale » (2014), tente de trouver sa place dans les travaux sur la régionalisation avancée et l'appel à des systèmes de gouvernance plus participatifs et démocratiques. Dans le contexte de la gestion de l'eau, cela s'est traduit par un découpage institutionnel des territoires en bassins hydrauliques et par une attention accrue donnée aux moyennes et petites exploitations ainsi qu'aux acteurs de terrain, si longtemps désavantagés par une approche moderne fondée sur les grandes infrastructures et les technologies. Se demander quels sont les biens communs « comptent le plus » nous amène à notre point focal : l'eau. La considérer comme le bien commun par excellence, mais également l'appréhender à travers une approche patrimoniale en revalorisant les savoir-faire dans un contexte cette fois de modernité pourrait aider à faire avancer le débat sur les types de développement alternatifs les plus appropriés.

\section{La modernité comme élément de " dé-communisation » du patrimoine et d'appropriation des communs}

Les approches économiques retenues dans la gestion d'un pays déterminent d'une façon assez marquée son identité et l'image que d'autres peuvent s'en faire. Pendant longtemps, le Maroc s'est présenté au reste du monde comme mariant traditions et modernité, une expression qui, dans le contexte de la gestion de l'eau, s'est avérée assez troublante. Beaucoup de pratiques traditionnelles ont été étouffées et délaissées au profit de la Grande Hydraulique ${ }^{4}$ depuis l'indépendance du pays. Comme l'explique Muriel Maillefert (2008), dans la zone de Marrakech, par exemple, l'ancien système des khettaras $^{5}$, pourtant efficace, a été mis à mal par la conjugaison de l'oubli de leur existence et des politiques d'aménagement et d'urbanisation.

$17 \mathrm{Au}$ lendemain du protectorat (1912-1956), le pays neuf et indépendant s'enorgueillit d'une modernisation rapide, dans la continuité cependant de ce que les autorités françaises avaient entrepris, centrée sur la transformation du pays en une économie agricole d'exportation. La construction de grands barrages ${ }^{6}$, les transferts ${ }^{7}$ d'eau entre 
régions, la mécanisation de l'agriculture et le morcellement des terres, se sont faits au détriment des terres montagneuses, à la fois négligées et privées de leur eau par les exploitations agricoles des plaines. Plus important encore, les communautés rurales ont été divisées et celles des zones montagneuses, appauvries, ont quitté en masse les campagnes, venant grossir la population des villes. Les changements juridiques qui avaient permis aux autorités protectorales d'exploiter des terres et qui allaient à l'encontre du droit coutumier de l'eau ont perduré après l'Indépendance, bénéficiant aux grandes familles qui avaient hérité des grandes exploitations agricoles. Ces divisions territoriales avaient déjà été opérées avant le protectorat avec la création de domaines de rentes, phénomène largement amplifié avec la modernisation de l'économie du pays et la montée du capitalisme agraire.

18 Le pays, bien qu'indépendant, n'en est pas moins désuni dans sa gestion des ressources. En premier lieu, l'eau du ciel, considérée comme un « don de Dieu » en Islam et devant être gérée, partagée et distribuée selon des codes traditionnels très précis, s'est trouvé régie par les nouvelles dispositions juridiques imposées par l'administration du protectorat français, elle-même accompagnée de groupes puissants de Marocains associés au Makhzen et impliqués dans les actions entreprises par les forces coloniales. En second lieu, l'eau qui était précédemment utilisée avec modération par une agriculture de subsistance dans une logique socio-écologique répondait à un besoin de survie et non pas à une logique d'enrichissement monétaire. En troisième lieu, cette nouvelle logique affecta la perception même du territoire. Alors que les tribus du Maroc avaient appris au long des siècles à vivre avec la rareté de l'eau et les aléas climatiques, le protectorat introduisit la distinction entre un Maroc " utile » et un Maroc " inutile ", négligeant les diversités géographiques et sociales du pays. Ce découpage en « espaces fractionnés » créé par le premier résident général du protectorat français, Hubert Lyautey, a été maintenu jusqu'à ce qu'on introduise, dans les années 1990, les notions d'aménagement du territoire (Mohaine 2017) et de régionalisation avancée.

$\mathrm{Au}$ tout début de l'indépendance, le territoire n'est donc pas considéré comme un commun : il est véritablement découpé par le choix d'une gestion moderne du pays. Qu'en est-il de l'eau? Sous le protectorat comme dès après l'indépendance, l'eau est consacrée à l'irrigation des cultures d'exportation - la surexploitation des nappes phréatiques à laquelle elle conduit est même encouragée par l'État. Comme pour les territoires auxquels elle est intimement liée, l'eau n'est donc pas considérée comme un bien commun. Sa privatisation et le désengagement de l'État suivent, dans les années 1980, les recommandations de la Banque mondiale, transformant les biens et services collectifs qui se rattachent à l'eau en marchandise. Cette réponse initiale à de sévères années de sécheresse à travers une gestion déléguée ${ }^{8}$ est très mal vécue par la société civile qui appelle à rétablir le droit d'accès à l'eau pour tous par la remunicipalisation de sa gestion. L'objectif est qu'au bout du compte, l'eau, bien vital, soit de nouveau considérée comme le bien public par essence dont la collectivité ne peut céder au marché ni la production, ni la préservation, ni la distribution et la gestion (El Menouar 2012: 87). Comme le souligne Mehdi Lahlou, directeur de l'Association contre la marchandisation de l'eau (Acme): "Nous considérons que ses caractéristiques de rareté, d'absolue nécessité et de non-substituabilité mettent l'eau dans l'échelle des besoins à satisfaire chez les hommes à une place à part, sans commune mesure avec aucun bien ou service [...]. Le non-respect manifeste d'un ensemble d'engagements contractuels majeurs de la part d'une société privée délégataire de services publics [...] rappelle que l'eau est par essence un bien public » (Lahlou 2010). On assiste donc à un 
retour en force de la société civile, à travers la création, notamment, d'associations, la participation à des débats nationaux sur la question de l'eau et sur l'amendement de la loi sur l'eau (ONSSA 2016), l'encouragement à mettre en place de meilleurs systèmes d'information dans ce domaine. Cette mobilisation est un appel à conférer une valeur de bien commun à l'eau et suscite un nouvel intérêt pour le patrimoine qu'elle représente.

Sous le protectorat, la notion de patrimoine de l'eau est, sinon inexistante, du moins ignorée, et non sans raison. Comme le souligne Paul Claval, « partout où s'étendit (la) mainmise coloniale, la question se posa de savoir ce qu'il convenait de faire de ce qui apparaissait comme un patrimoine. Si on choisissait de le conserver, sur quels critères retenir les ensembles à préserver " (Gravari Barbas \& Anguis $2003: 45)$. Les approches muséales encouragées par le bureau des affaires indigènes au Maroc ne s'intéressaient pas aux savoir-faire locaux en matière de gestion d'un milieu naturel que les communautés locales connaissaient pourtant mieux que quiconque. Les modes de production agricole introduits sous le protectorat, modernes et nouveaux, devaient pouvoir remplir les objectifs fixés à l'échelle du pays, lesquels étaient hors d'atteinte par les approches traditionnelles. De ce fait, les cultures vivrières ont été totalement bouleversées et négligées, pour ne pas dire méprisées et considérées comme archaïques, aussi "inutiles» que les régions ne permettant pas de produire des cultures d'exportation. La vulnérabilité du patrimoine, et des savoir-faire traditionnels de la gestion de l'eau, en particulier, s'est accrue du fait que les changements législatifs mis en place au cours du protectorat et maintenus en grande partie après l'indépendance, défendaient un modèle de gestion de la ressource, fondé sur la Grande Hydraulique et l'irrigation, non conforme aux principes traditionnels des petites exploitations d'agriculture de subsistance.

21 Il est clair que si la difficulté principale dans la gestion du patrimoine réside dans la capacité à s'entendre sur la valeur de ses éléments pour pouvoir décider ce qui doit être préservé, ce qui doit être transformé et ce qui doit être détruit, « il faut commencer par se demander pour qui ces éléments ont de la valeur » (Thérond \& Trigona 2009: 8). Il est également certain que les savoir-faire relatifs à la gestion de l'eau en milieu aride ou semi-aride ont une valeur unique aux yeux des populations locales dont la survie dépend de leur résilience dans un milieu naturel hostile. Pour les autorités coloniales puis de celles qui leur ont succédé lors de l'Indépendance, ce « patrimoine » n'était pas encore considéré comme une richesse. Ce n'est qu'aujourd'hui que, la valeur de l'eau en tant que bien commun et des techniques aidant à en gérer la rareté étant mieux comprise, on cherche à préserver, voire à ranimer des pratiques moribondes, souvent sur le point de disparaitre. Le discours sur l'économie du patrimoine, sur la médiation patrimoniale et sur la patrimonialisation offre peut-être à ces savoir-faire une chance de survie. La prise de conscience des tensions sociales et des inégalités économiques et régionales peut aussi aider à ré-appréhender les dimensions sociales de la gestion de l'eau au Maroc. Le défi auquel fait face un tel pays est donc de ne plus considérer les savoir-faire traditionnels de gestion de l'eau comme n'appartenant qu'à certaines régions isolées mais comme un véritable patrimoine immatériel précieux et utilisable par tous et partout, dans un souci de préservation systémique d'un bien commun naturel absolument vital pour pouvoir atteindre les objectifs économiques fixés au plan national. 


\section{Identité, culture et milieu naturel : les biens communs liés au patrimoine}

On assiste donc - un peu in extremis - à une redécouverte de ces savoir-faire, en partie encouragée par les approches environnementalistes participatives qui se multiplient à l'échelle mondiale (Kato, Nakagami \& Cooper 2016) et démontrent que les experts sont ceux qui connaissent le plus intimement le terrain et ses spécificités (les paysans) et que partir de la base plutôt que du sommet permet d'impliquer les participants dans les projets et de leur accorder la place qui leur revient dans la conduite et l'entretien de ces projets.

Ce changement est en partie dû aux agitations d'une société civile ${ }^{9}$ et, d'une façon moindre, du monde rural, qui inquiètent les autorités. Il tient aussi à la redécouverte de la ressource vitale qu'est l'eau, et des questionnements concernant les meilleures façons de la gérer. Le plan national de l'eau de 2001, la stratégie nationale de l'eau de $2009^{10}$ ainsi que la nouvelle loi sur l'eau de 2016 (ONSSA 2016) témoignent de l'intérêt porté spécifiquement à ce bien commun, et non exclusivement à ce qu'il permet de créer (Tazi Sadeq 2006).

Le Maroc s'est récemment engagé dans un débat autour de ces modes alternatifs de développement. Déjà officiellement impliqué dans la défense du développement durable, le pays s'est lancé dans de grands projets sur les énergies renouvelables ${ }^{11}$. Il cherche à réduire sa dépendance énergétique, à exporter de l'électricité mais aussi à occuper une place de premier rang dans un certain nombre de thématiques à l'échelle de l'Afrique. Du point de vue de la gestion de l'eau, beaucoup reste à faire pour choisir la voie la plus appropriée et durable. La redécouverte de son propre patrimoine pourrait entrer dans cette logique de différenciation.

En période de stress hydrique, on tend à être plus sensible aux exemples de pratiques qui favorisent la résilience et l'adaptation. Ainsi a-t-on pris conscience de la capacité d'innovation du monde rural, elle-même associée à un riche héritage de savoirs. "Le monde rural marocain, c'est une culture faite tout à la fois de la connaissance du milieu naturel, des savoir-faire, des langues, de l'histoire locale, des traditions sociales, des comportements en société, des fêtes et des façons de vivre la religion " (Lazarev \& Naciri 2014 : 21). 


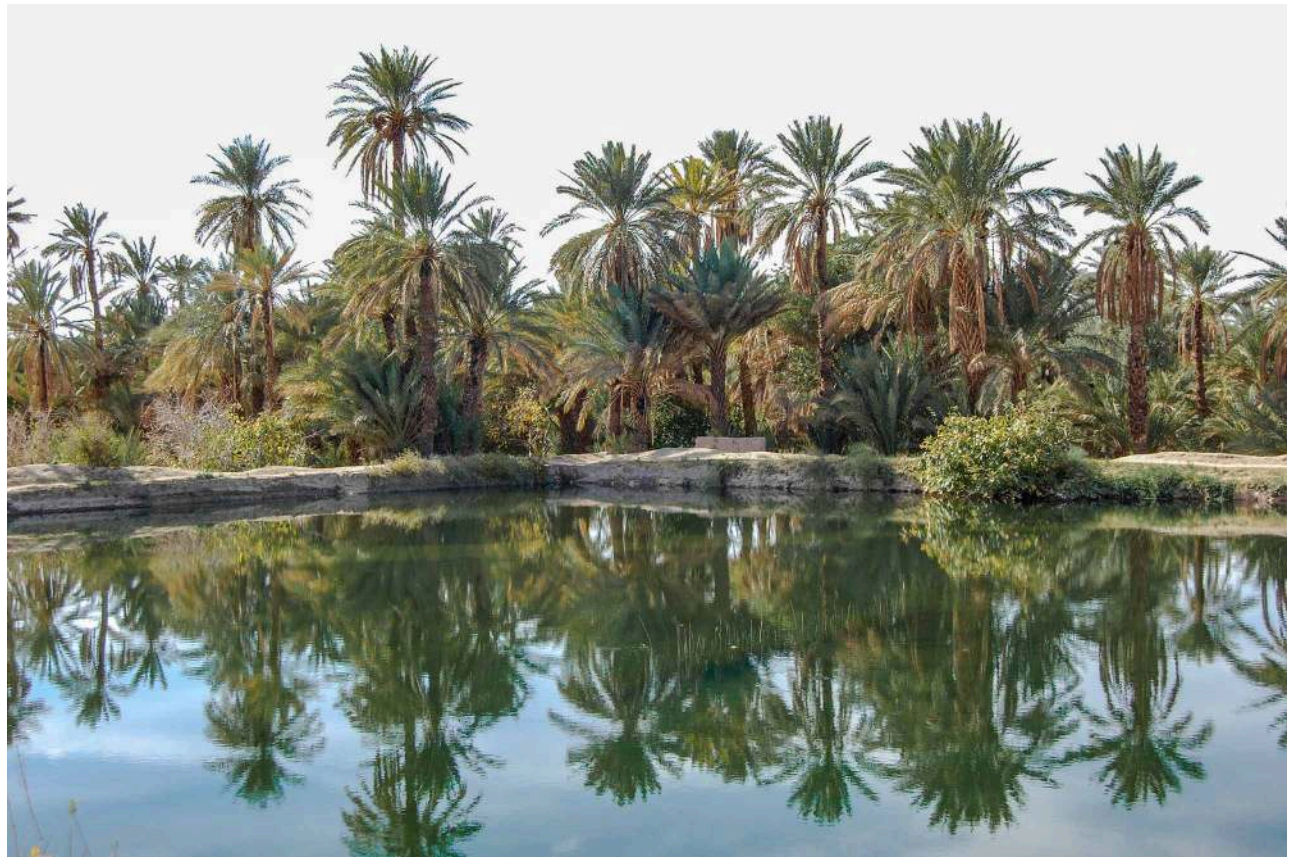

Capturer l'eau de pluie est un bon moyen de surmonter les problèmes de pénuries, surtout dans les régions oasiennes. Réservoir d'eau dans le sud marocain.

(c) Photo Sandrine Simon.

Le recours opéré par le Maroc à la richesse et l'ingéniosité des civilisations hydrauliques nous permet de voir réactivées tout un éventail de techniques héritées à la fois des apports romains et grecs mais aussi et surtout moyen-orientaux par l'intermédiaire des agronomes d'Al Andalous, du temps de l'occupation maure dans le sud de l'Espagne. Ces apports peuvent être regroupés en techniques agricoles, en modes de gestion des conflits et de partage de l'eau et en choix architecturaux et urbanistiques (Simon 2020). Ainsi, par exemple, les norias, roues hydrauliques qui permettent de puiser l'eau et d'irriguer les champs alentour, venaient à l'origine du Moyen-Orient, berceau de l'âge d'or des sciences du monde arabe entre le $\mathrm{IX}^{\mathrm{e}}$ et le $\mathrm{XIII}^{\mathrm{e}}$ siècle. De même, les khettaras, galeries souterraines permettant de transporter l'eau sur de grandes distances en évitant son évaporation, avaient été inventées en Iran. C'est grâce aux khettaras, que la ville de Marrakech a pu s'établir jusqu'à devenir une capitale.

Le réseau des séguias, système traditionnel de distribution de l'eau de surface en milieu rural encore utilisé, sert à partager l'eau d'irrigation tout en suivant des codes de répartition bien établis et gérés par la communauté. Les gens se sont rendu compte que les nouvelles séguias construites en ciment n'étaient pas aussi efficaces que celles, traditionnelles, construites en terre, qui permettaient à l'eau de s'infiltrer tout en s'écoulant. La "perte », pouvant atteindre jusqu'à $50 \%$ de l'eau transportée, assurait qu'elle s'écoule encore en aval, le droit coutumier donnant la priorité aux utilisateurs de l'amont sans pour autant défavoriser les autres utilisateurs. 
Figure 6
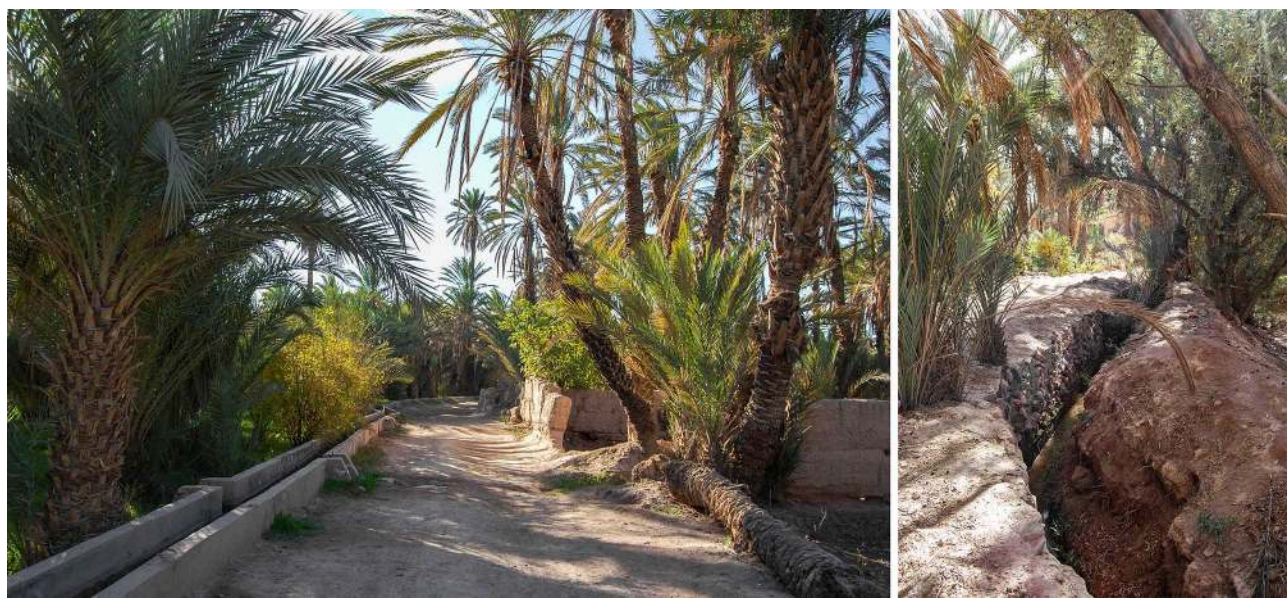

Les séguias en ciment sont en train de remplacer les séguias en terre bien que ces dernières permettent une meilleure infiltration de l'eau sur leur trajet, et une plus grande collecte de l'eau restante après irrigation par les fermiers en aval. À gauche : séguia en ciment. À droite : séguia en terre.

(c) Photos Sandrine Simon. le statut de certaines pratiques de gestion de l'eau dans d'autres pays. Ainsi, les savoirfaire des mesureurs d'eau des foggaras (système d'irrigation), ou aiguadiers, des communautés ksouriennes du Touat et du Tidikelt en Algérie ont été inscrits ${ }^{13}$ sur la Liste du patrimoine immatériel nécessitant une sauvegarde urgente par l'Unesco en 2018. 


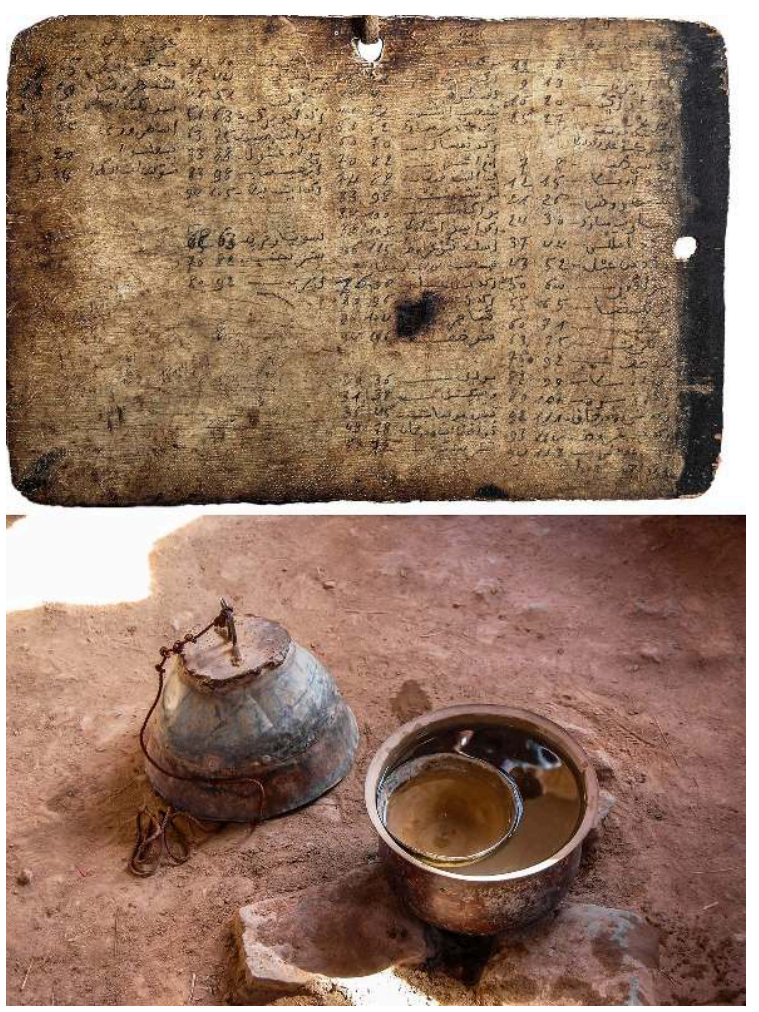

La distribution de l'eau pour l'irrigation des champs est basée sur le temps : les familles bénéficiant de l'irrigation bénéficient d'une certaine durée d'irrigation, mesurée par l'horloge. Le pot met quarantecinq minutes à se remplir. À la fin d'une durée d'irrigation, l'eau de la seguia est déviée vers un autre champ. En haut : liste des familles bénéficiant de l'irrigation mesurée. En bas : horloge à eau pour mesurer l'unité d'irrigation par unité de temps.

(c) Photos Sandrine Simon.

31 C'est le signe d'un élan manifeste en faveur d'une protection des biens communs matériels ainsi que du patrimoine matériel et immatériel en Afrique du Nord et plus spécifiquement au Maroc.

\section{Vers des modes de développement alternatif : une nouvelle politique de l'eau basée sur la protection des communs par le biais de la protection du patrimoine}

Ces dernières années, un travail considérable de sensibilisation de la population du Maroc à la question de l'eau a été mené. Le rappel de son importance vitale en tant que bien commun fondamental s'accompagne d'une approche patrimoniale dans la mesure où le débat qu'elle suscite, dans le contexte marocain, semble aller immanquablement de pair avec celui que soulève la question des savoir-faire en matière de gestion de l'eau, véritables piliers des civilisations hydrauliques. Ces pratiques ont été mises en valeur dans le musée pour la civilisation de l'eau, créé à Marrakech il y a quelques années.

33 À Fès, cette valorisation du bien commun est aussi passée par celle du patrimoine, en l'occurrence celui que constitue la fameuse horloge hydraulique datant du XIV siècle, faisant actuellement l'objet d'études par les autorités qui prévoient de la réhabiliter. 
Dans cette même ville, le Forum de débats accompagnant le festival international annuel des musiques sacrées était consacré, en 2017, au thème " L'eau et le sacré14 ».

Figure 8

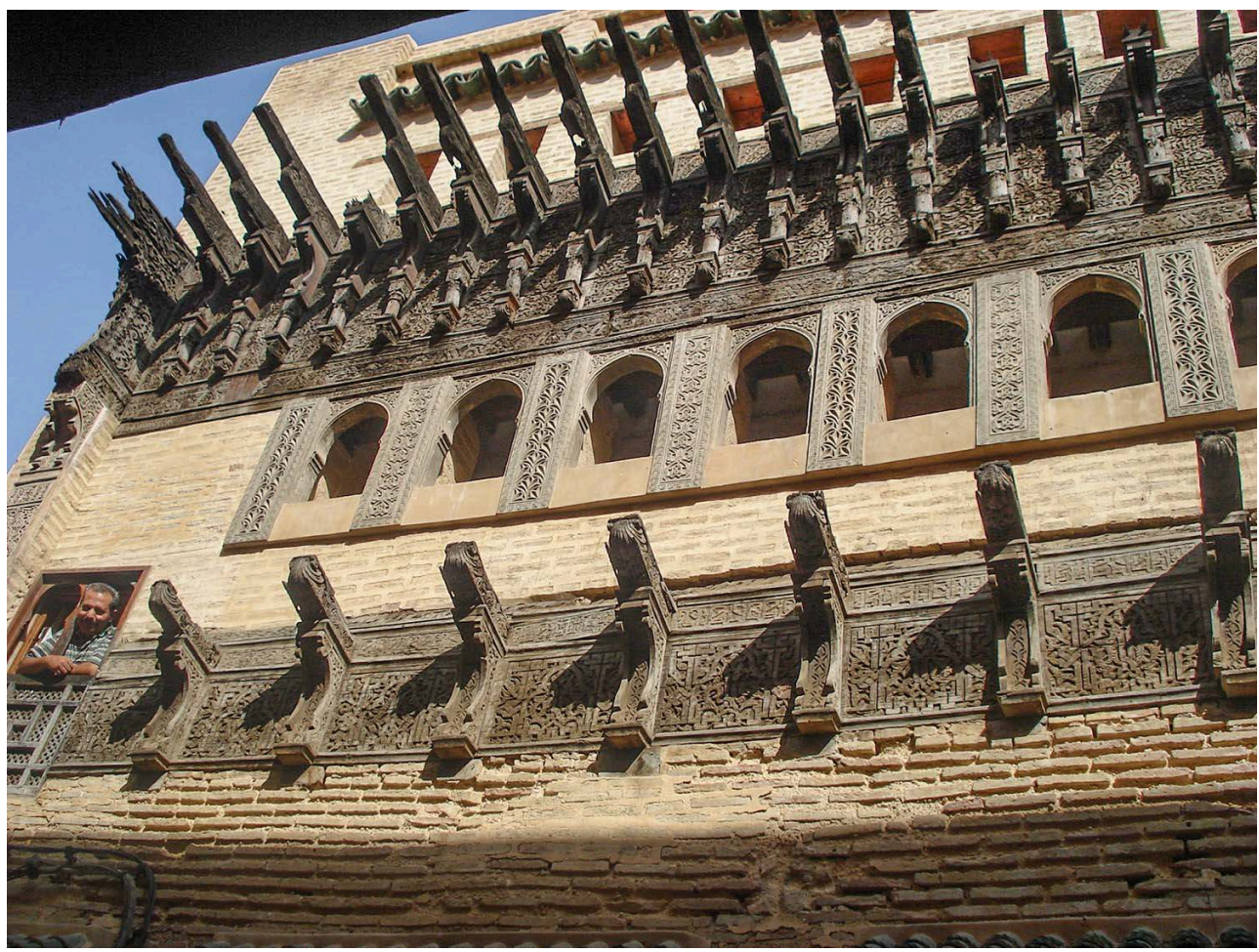

L'horloge à eau construite par la dynastie des Mérinides à Fès était aussi performante qu'esthétique et décorative. Située dans une des ruelles principales de la vieille ville, en face d'une des mosquées les plus célèbres du quartier, la Bouananya, elle témoigne du prestige de cette dynastie.

(c) Photo Sandrine Simon.

Mais l'approche patrimoniale qui semble actuellement s'éveiller va bien plus loin. En accord avec la politique de régionalisation avancée, la gestion patrimoniale recommande de créer un nouveau "bien commun" entre des acteurs aux représentations et aux logiques différentes (attachées à un bassin hydraulique ou à une région, par exemple) et aussi d'instaurer un processus de négociation leur permettant d'élaborer une charte de compromis à long terme. Or, pendant longtemps au Maroc, le monde rural a été considéré comme devant faire l'objet d'un contrôle et d'un encadrement, donc, dans la logique des autorités, de vigilance et de suspicion. Aussi ce changement politique est-il un tournant majeur. La capacité associative du monde rural doit être respectée: les institutions coutumières telles que les jmaa villageoises qui existent depuis des siècles méritent ainsi sans doute d'être mieux comprises plutôt que remplacées par des associations d'usagers imposés "par le haut ». Comme le souligne Cominelli (2012: 89), « inclure les savoir-faire traditionnels permet donc de sensibiliser les décideurs publics et d'établir des modèles alternatifs de gestion, ni publics, ni privés. [De plus], mettre en avant la notion de bien commun peut aider les membres d'une communauté à comprendre qu'ils ne sont pas seulement des consommateurs passifs, mais que leur engagement est sollicité pour une gouvernance appropriée de leur patrimoine ». 

possible de conjuguer l'efficacité (les ressources ne sont pas épuisées mais conservées ou renouvelées) et l'équité (dans le partage des ressources et le mode de règlement des conflits). Les modes de gestion collective de l'eau pratiqués en agdals ${ }^{15}$ par les institutions coutumières du Haut Atlas marocain dans la vallée des Aït Bouguemez incarnent une success story au regard de la liste de critères favorables identifiés dans les travaux fondateurs d'Ostrom. L'approche patrimoniale souhaite faire émerger de ces modes de gestion un processus cognitif qui permettra de les adapter dans le long terme.

Figure 9

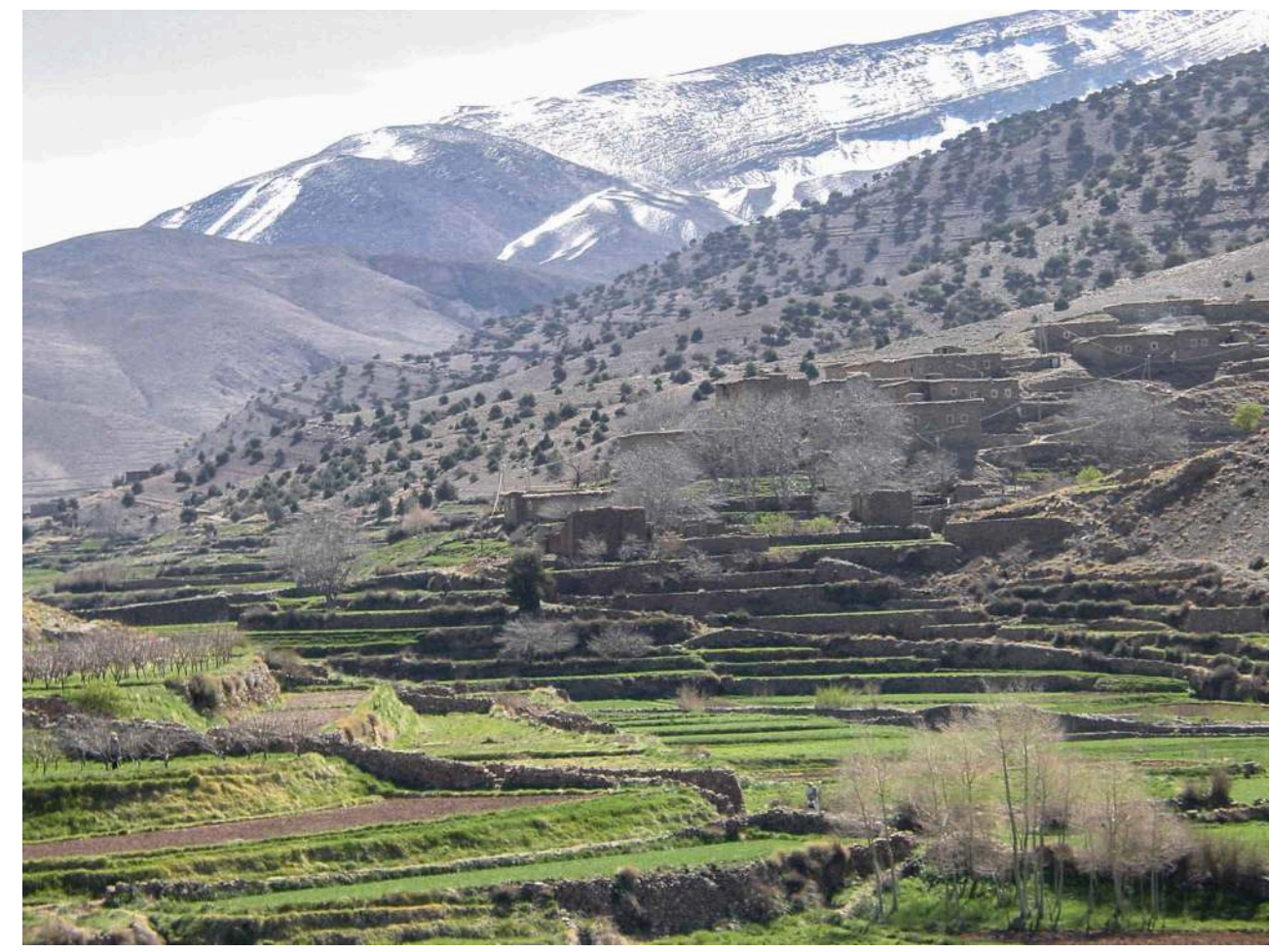

Cultures en terrasses dans la vallée des Ait Bouguemez. L'adaptation des communautés berbères à un milieu montagneux a résulté en l'établissement de terrasses, irriguées par séguias et eau de source. La vallée a conservé des institutions locales vivantes intervenant dans la gestion des ressources renouvelables communes (eau, forêts, pâturages).

(c) Photo Didier Genin, distr. IRD (http://www.indigo.ird.fr)

D'un point de vue technique, comment les savoir-faire traditionnels peuvent-ils être réintégrés dans des pratiques plus modernes? Alors que jusqu'à présent, les nouveaux projets de développement avaient du mal à se greffer sur les modes de gestion communautaire des ressources préexistants, de nouvelles initiatives sont prises pour créer des alliances entre les différentes pratiques mais aussi entre acteurs. Ainsi par exemple, le savoir-faire des khatatiriya, constructeurs des khetarras, était sur le point d'être perdu lorsque, dans les années 1990, afin d'encourager l'exploitation de toute source possible d'eau, les gouvernements locaux de certaines régions ont reçu des aides financières pour assurer la maintenance des khetarras encore existantes (Belhouari 2017). Par ailleurs, des chercheurs de l'IRD ${ }^{16}$ (Roose, Sabir \& Laouina 2010) ont mis en place une stratégie de " gestion conservatoire de l'eau et des sols » (GCES). Leur objectif est de transmettre un savoir paysan, analysé dans l'optique d'une approche scientifique de la gestion des ressources naturelles en milieu méditerranéen, et plus 
particulièrement au Maroc, afin d'éviter que ces connaissances ne soient à jamais perdues. Ils ont répertorié un ensemble de savoir-faire et de régions dans lesquelles on pourrait mettre en œuvre une approche patrimoniale de l'eau.

Figure 10
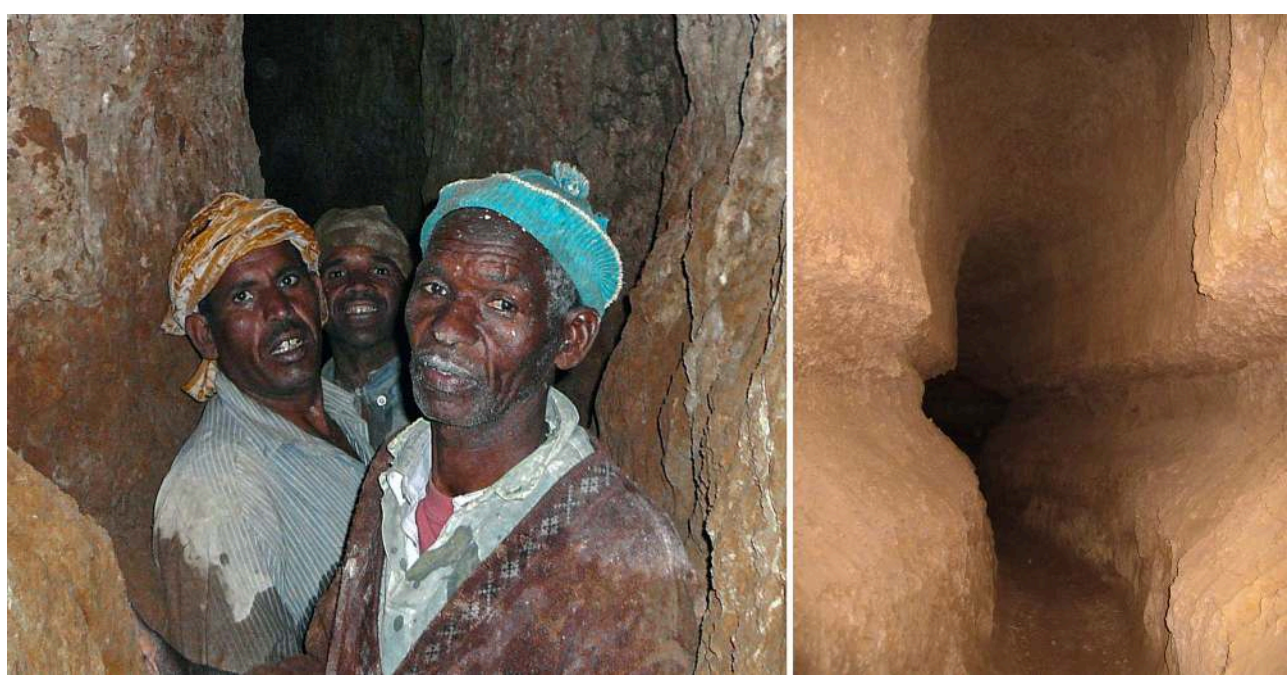

Les khetarras, galeries souterraines permettant le transfert d'eau de régions riches en eau a des régions plus arides, utilise le principe de la gravite et évite toute évaporation et détérioration de la qualité de l'eau. À gauche : les villageois entretiennent les khettaras ou galeries drainantes (Tafilalet, Maroc). À droite : galerie souterraine de khettara (Tafilalet, Maroc).

(c) Thierry Ruf, distr. IRD (http://www.indigo.ird.fr)

Le potentiel de réintégration des savoir-faire traditionnels, pour des raisons techniques, économiques, de gouvernance, culturelles et sociales, est donc considérable et prometteur. Le répertoire de l'ensemble de ces initiatives (Simon 2020) montre que la renaissance des savoir-faire locaux pourrait assurer la sauvegarde des ressources en eau.

\section{Conclusion}

Au Maroc, la situation de stress hydrique croissant pousse les dirigeants à envisager de nouveaux modes de gestion de l'eau plus adaptés aux contraintes actuelles. La mise en œuvre d'une approche patrimoniale, envisagée depuis peu seulement, permet une interaction entre les dimensions écologiques, économiques et sociales que ces nouveaux modes de gestion devraient consolider afin de respecter les objectifs de développement durable du pays. Le bilan des observations de ces approches nous permet de conclure sur quelques points importants qui pourraient élargir notre compréhension non seulement des notions de patrimoine et de communs, mais aussi des liens forts qui les unissent. Ceci pourrait nous aider à appréhender leur intégration dans des stratégies de développement considérant que la prospérité écologique et sociale est au fondement de la prospérité économique et de la stabilité politique.

Le patrimoine de l'eau intègre et dépasse d'une certaine façon la notion de communs : le patrimoine de l'eau, c'est la relation permanente et vivante des communautés qui coexistent avec cette ressource naturelle qu'elles utilisent, qu'elles protègent et dont leur survie dépend. Cette relation est donc d'ordre économique, écologique, mais aussi 
technique et même spirituelle. Les savoir-faire et infrastructures traditionnels qui participent à la gestion de l'eau existent parce que, fondamentalement, l'eau est reconnue comme le don de dieu, la ressource face à laquelle les hommes sont égaux.

Figure 11

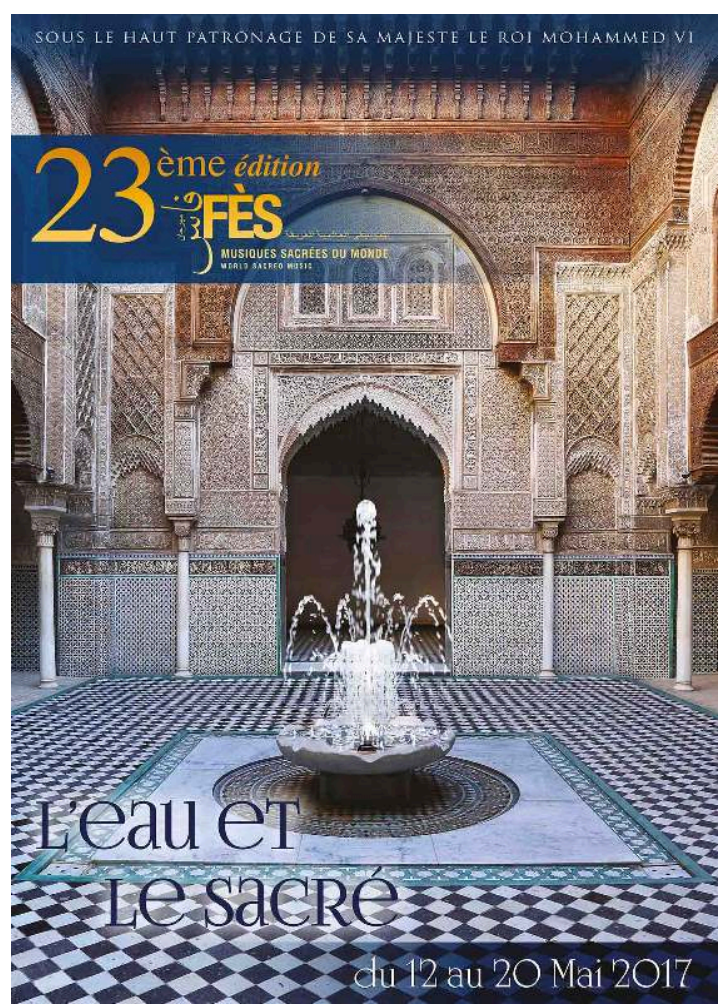

En Islam, l'eau est symbole de vie ainsi que du paradis. En 2017, elle a constitué la thématique principale du Festival des musiques sacrées du monde, qui a lieu à Fès, capitale spirituelle du Maroc.

Reproduction Festival des musiques sacrées du monde. encore l'accès inégal à l'eau potable. Les pratiques économiques modernes qui ont marginalisé les pratiques patrimoniales ont rompu l'unité des communautés humaines face à l'une de leurs ressources communes les plus précieuses : l'eau.

41 Mais le nouveau discours tenu sur la protection de l'environnement et l'impératif absolu de trouver des solutions pour gérer l'eau de façon plus efficiente offrent le moyen de valoriser les savoir-faire ancestraux et résilients et d'inclure les communautés détentrices de ces savoir-faire dans des modes de gestion conformes à de nouveaux modes de développement et de gouvernance. Cette inclusion sera peut-être d'autant plus réussie que le patrimoine culturel matériel et immatériel de l'eau est introduit dans le discours politique comme capital social à protéger au même titre que le capital naturel. Ce faisant, le partenariat capital social-naturel-économique pourrait peut-être permettre d'atteindre l'objectif premier de sauvegarde de ce commun.

Dans un contexte politique où la régionalisation « avancée " progresse lentement... et où le choix de l'inclusion de communautés précédemment marginalisées est délicat, la logique de protection du patrimoine pour sauvegarder l'eau est défendable. Ainsi, la notion de patrimoine pourrait elle aussi être revisitée grâce à une réflexion sur la notion de communs. Une post-modernité, favorable à l'intégration du patrimoine culturel 
immatériel à de nouvelles technologies, après une modernité génératrice de cassures des biens communs et des patrimoines, réconcilierait enfin société civile et gouvernement avec les notions de protection du bien commun par excellence grâce à la redécouverte du patrimoine de l'eau.

\section{BIBLIOGRAPHIE}

BELHOUARI Sabrina, 2017, « Les khettaras du Tafilalet, un patrimoine précieux à préserver ", L'Économiste.com [en ligne], nº 5086, https://www.leconomiste.com/article/1016222-leskhettaras-du-tafilalet-un-patrimoine-precieux-preserver [lien valide en janvier 2021].

BROMLEY Daniel W., 1992, Making the commons work. Theory, practice and policy, San Francisco, Institute for Contemporary Studies Press.

COMINELLI Francesca, 2012, « Le patrimoine culturel immatériel est-il un bien commun ? Le cas de la pierre sèche en France ", Revue de l'organisation responsable, vol. 7, p. 83-92. Disponible en ligne, https://www.cairn.info/revue-de-l-organisation-responsable-2012-2-page-83.htm [lien valide en janvier 2021].

CONSEIL DE L'EUROPE, 2005, Framework convention on the value of cultural heritage for society / Convention-cadre du Conseil de l'Europe sur la valeur du patrimoine culturel pour la société, Faro, Conseil de l'Europe, série « Traités du Conseil de l'Europe », n 199. Disponible en ligne, https://rm.coe.int/CoERMPublicCommonSearchServices/DisplayDCTMContent? documentId=0900001680083744 [lien valide en janvier 2021].

THÉROND Daniel \& TRIGONA Anna (dir.), 2009, Le Patrimoine et au-delà, Strasbourg, Éditions du Conseil de l'Europe. Disponible en ligne, https://rm.coe.int/ CoERMPublicCommonSearchServices/DisplayDCTMContent?documentId=09000016806abdeb [lien valide en janvier 2021].

EL FAÏZ Mohammed, 2001, « Le modèle de la Grande Hydraulique dans le Haouz de Marrakech », in GOBE Éric (dir.), Les Ingénieurs maghrébins dans les systèmes de formation, Tunis, Institut de recherche sur le Maghreb contemporain, coll. « Études et travaux de l'IRMC ». Disponible en ligne, p. 213-225. https://books.openedition.org/irmc/149 [lien valide en janvier 2021].

EL MENOUAR Allal, 2012, Pour une gouvernance optimale de l'eau au Maroc, Casablanca, Imprimerie Bidaoui.

FAOUZI Hassan, 2011, «L'Agdal dans la dynamique des systèmes agraires des arganeraies des Haha (Haut-Atlas occidental, Maroc) », Études caribéennes, $\mathrm{n}^{\circ} 20$, « Tourisme, culture(s) et attractivité des territoires » [en ligne], https://journals.openedition.org/etudescaribeennes/5569 [lien valide en janvier 2021].

FIORENTINO Paola, FRIEL Martha, MARELLI Massimo \& SANTAGATA Walter, 2010, Cultural commons and cultural communities. The case studies of Milan designers and Italian Futurists artists, Turin, Centro Studi Silvia Santagata (Dipartimento di Economia S. Cognetti de Martiis, International Centre for Research on the Economics of Culture, Institutions, and Creativity), coll. « Working paper new series ». Disponible en ligne, https://www.fondazionesantagata.it/wpcontent/uploads/2_WP_Ebla_CSS-2.pdf [lien valide en janvier 2021]. 
GRAVARI-BARBAS Maria \& GUICHARD-ANGUIS Sylvie, 2003, Regards croisés sur le patrimoine dans le monde à l'aube du XXI siècle, actes de colloque (Paris, 7-9 octobre 1999), Paris, Presses de l'université de Paris-Sorbonne, coll. « Asie \& géographie ».

KATO Hisaaki, NAKAGAMI Ken'ichi \& COOPER Malcom, 2016, « Participatory approaches to environmental management. Future design for water resources management », in NAKAGAMI Ken'ichi, KUIBOTA Jumpei \& SETIAWAN Budi Indra, Sustainable water management. New perspectives, design and practice, Singapore, Springer.

LAHLOU Mehdi, 2010, « La privatisation de l'eau au Maroc : premiers constats à partir de l'expérience de la Lyonnaise des Eaux à Casablanca », Partage des eaux [en ligne], https:// www.partagedeseaux.info/La-privatisation-de-l-eau-au-Maroc-premiers-constats-a-partir-de-lexperience [lien valide en janvier 2021].

LASSERRE Frédéric, 2009, Écologie, irrigation, diplomatie, comment éviter les guerres de l'eau. L'eau au coeur des conflits $d u x^{e}$ siècle, Paris, Delavilla.

LAZAREV Grigori (dir.), 2000, Vers un éco-développement participatif. Leçons et synthèse d'une étude thématique, Paris, L'Harmattan, coll. « Bibliothèque du développement ».

LAZAREV Grigori \& NACIRI Mohamed, 2014, Ruralité et changement social. Études sociologiques, Rabat, Publications de la faculté des Lettres et Sciences humaines, coll. « Essais et études ».

MAILLEFERT Muriel, 2008, «La “mise en patrimoine” de l'eau : une perspective interdisciplinaire. Compte rendu des journées d'Arras 9-10 mars 2007 ", Natures Sciences Sociétés, vol. 16, n 3, «Varia », p. 282-284. Disponible en ligne, https://www.cairn.info/revue-natures-sciencessocietes-2008-3-page-282.htm?contenu=resume [lien valide en janvier 2021].

MOHAINE Abdelkader, 2017, La Géographie et l'aménagement au Maroc : regards croisés, Casablanca, Afrique Orient.

MICHON Perrine (dir.), 2019, Les Biens communs. Un modèle alternatif pour habiter nos territoires au XXI siècle, textes issus de la journée d'étude « Du bien commun aux biens communs: formes, gouvernance et acteurs d'une politique territoriale autour de la question du/des bien(s) commun(s)» (17 février 2016), Rennes, Presses universitaires de Rennes.

ONSSA, 2016 (6 octobre), « Dahir no.1-16-113 du 6 kaada 1437 portant promulgation de la loi 36-15 relative à l'eau », Bulletin officiel, n 6506.

OSTROM Elinor, 1990, Governing the commons. The evolution of institutions for collective action, Cambridge, Cambridge University Press.

PASSET René, 1990, « Environnement et biosphère », in GREFFE Xavier, MAIRESSE Jacques \& REIFFERS Jean-Louis (dir.), Encyclopédie économique, Paris, Economica, p. 1815-1868.

PETIT Olivier \& ROMAGNY Bruno, 2009, «La reconnaissance de l'eau comme patrimoine commun : quels enjeux pour l'analyse économique?", Mondes en développement, $\mathrm{n}^{\circ}$ 145, vol. 1 , « La mise en patrimoine de l'eau », p. 29-54. Disponible en ligne, https://www.cairn.info/revuemondes-en-developpement-2009-1-page-29.htm [lien valide en janvier 2021].

PETIT Olivier, 2015, « L'eau douce : bien(s) commun(s) ou patrimoine ? », in EUZEN Agathe, JEANDEL Catherine \& MOSSERI Jeremy (dir.), L'Eau à découvert, Paris, CNRS éditions, coll. « À découvert ».

PETITJEAN Olivier, 2016, « Introduction du dossier : les guerres de l'eau auront-elles lieu ? », Partage des eaux [en ligne], https://www.partagedeseaux.info/Introduction-du-dossier-lesguerres-de-l-eau-auront-elles-lieu [lien valide en janvier 2021]. 
ROOSE Éric, SABIR Mohammed \& LAOUINA Abdellah, 2010, Gestion durable de l'eau et des sols au Maroc. Valorisation des techniques traditionnelles méditerranéennes, Marseille, IRD.

SIMON Sandrine, 2020, Histoire d'eau. Le patrimoine de l'eau jaillie des sources pour un développement alternatif au Maroc, Paris, L'Harmattan, coll. « Harmattan le Grand Maghreb ».

TAZI SADEQ Houria, 2006, Du droit de l'eau au droit à l'eau au Maroc et ailleurs, Casablanca, La croisée des chemins.

SAVAGE Thomas, BOUDARHAM Mohammed \& AHDANI Jassim, 2017, « La soif nous guette », TelQuel, $\mathrm{n}^{\circ}$ 783, numéro spécial, p. 33-45.

UNESCO, 2003, Convention pour la sauvegarde du patrimoine culturel immatériel, Paris, Unesco. Disponible en ligne, https://ich.unesco.org/fr/convention [lien valide en janvier 2021].

WADE Robert, 1988, Village republics. Economic conditions for collective action in South India, Cambridge, Cambridge University Press, coll. « Cambridge South Asian studies ».

\section{NOTES}

1. La régionalisation avancée qui vise à décentraliser les prises de décision a pris son réel élan à la fin des années 1990 au Maroc mais elle est sujette à controverse du fait des difficultés qu'elle rencontre dans sa mise en œuvre (Mohaine 2017).

2. Traduite en termes de quantité d'eau disponible par habitant par année, tels les $500 \mathrm{~m}^{3} /$ homme/an annoncés pour 2020 au Maroc par l'enquête du magazine TelQuel (Savage, Boudarham \& Ahdani 2017).

3. La thèse de Garrett Hardin démontrait que, face à une ressource limitée, le comportement humain conduit à l'exploiter à des fins individuelles et aboutit inévitablement à sa dégradation. Cette thèse a servi à justifier la privatisation des ressources comme modalité optimale de gestion. Elinor Ostrom a cependant démontré qu'une communauté d'individus peut adopter des modalités de fonctionnement permettant une gestion durable des ressources en satisfaisant à la fois les intérêts individuels et collectifs. Ses travaux relatifs à la gouvernance des communs lui ont valu le prix Nobel d'économie en 2009.

4. Modernisation de la gestion de l'eau à travers des projets d'infrastructures de très grande envergure (El Faïz 2001).

5. Système d'irrigation traditionnel qui consiste à exploiter la nappe phréatique grâce à une sorte de drain souterrain constitué par une succession de puits reliés entre eux. C'est l'un des procédés traditionnels utilisés dans la plaine du Haouz.

6. Le Maroc en compte maintenant dans les 144 dont 104 sont de "grands barrages ", selon la FAO (2005).

7. Voir l'article de Julie Chaudier (2016) paru dans Yabiladi,com en date du 4 mai 2016 : https:// www.yabiladi.com/articles/details/44349/autoroute-l-eau-travaux-devraient-commencer.html [lien valide en janvier 2021].

8. Contrat par lequel une personne morale de droit public, appelé le délégant, délègue pour une durée limitée un service public dont il a la responsabilité à une personne morale de droit public ou privé, appelé le délégataire, le droit d'en prélever le coût auprès des bénéficiaires ou réaliser des bénéfices ou les deux (El Menouar $2012: 88$ ).

9. Manifestations contre le prix de l'eau, par exemple (Savage, Boudarham \& Ahdani 2017).

10. Voir en ligne le résumé de cette stratégie publié par le Secrétariat d'État auprès du ministre de l'Énergie, des Mines et du Développement durable (Royaume duMaroc): http:// www.environnement.gov.ma/PDFs/EAU/resume.pdf [lien valide en janvier 2021]. 
11. Le complexe solaire Noor, composant principal du plan national marocain des énergies renouvelables, abrite la centrale Noor 1 , inaugurée en 2016 dont le coût total s'élève à 600 millions d'euros pour une capacité de 160 mégawatts. C'est la septième plus grande centrale solaire du monde.

12. Maison traditionnelle dont les pièces sont disposées et tournées vers un patio central avec jardin.

13. Voir https://ich.unesco.org/fr/USL/les-savoirs-et-avoir-faire-des-mesureurs-deau-desfoggaras-ou-aiguadiers-du-touat-tidikelt-01274 [lien valide en janvier 2021].

14. Voir la publication qui accompagnait le festival : FONDATION ESPRIT DE FÈS, 2017, Forum 2017. L'eau et le sacré, Fès, Les éditions de la Fondation Esprit de Fès.

15. Les agdals sont décrits comme une "pratique de gestion emblématique de la montagne berbère » par Hassan Faouzi (2011), spécialiste du sujet.

16. Institut de recherche et de développement.

\section{RÉSUMÉS}

Considérant les difficultés à gérer une ressource naturelle qui se fait de plus en plus rare du fait des changements climatiques mais aussi de systèmes de gestion inadaptés aux contraintes socioéconomiques et écologiques, cet article explore la façon dont, au Maroc, les notions de communs et de patrimoine peuvent être engagées dans un dialogue constructif. Celui-ci a pour objectif de faire émerger des modes de développement alternatif basés sur une approche systémique de l'eau dans un pays semi-aride. L'article révèle l'importance que ces concepts - mais aussi les débats que suscitent leur signification - peuvent avoir pour aider à dépasser les modèles de modernité instaurés après l'indépendance et opter pour des stratégies de protection des communs réellement prometteuses. Celles-ci gagneraient à être fondées sur un patrimoine qui, loin d'avoir été valorisé et considéré comme une richesse partagée, a longtemps été étouffé par une gestion moderne de l'eau basée sur la Grande Hydraulique et la marginalisation des milieux ruraux traditionnels. Le stress hydrique croissant et les nouvelles structures de gouvernance décentralisées appellent à une revalorisation des savoir-faire locaux, pratiques qui, tant sur le plan socioéconomique qu'écologiques, sont finalement les mieux adaptées aux contraintes actuelles.

The point of departure of this article is the difficulties in managing a natural resource whose scarcity is increasing as a result of climate change and of management practices that are illadapted to both ecological and socio-economic current constraints. The article explores how the notions of common resources and heritage can help us engage in a constructive reflexion on modes of alternative development based on systemic approaches of water resources in a semiarid country: Morocco. It demonstrates that these concepts can help to extend our economic reflexion beyond the concept of modernity introduced during and just after the French Protectorate that favored technological advances in agriculture and lead to the marginalization of rural communities and of their traditional know-how in agriculture and water management. Processes of advanced regionalization as well as the discontent of the civil society with regards to the social impacts emerging from the integration of a vital public good into the market sphere, have led to a new valorization process of water both as a public common good and as a precious heritage. 


\section{INDEX}

Mots-clés : développement durable, droit, eau, gestion et conservation, gouvernance patrimoniale, Maroc, mobilisation sociale, modernisation de l'agriculture, modernité, régionalisme, religion, savoir-faire, territoires, traditions, transmission des savoirs

Keywords : sustainable development, law, water, management and conservation, heritage governance, Morocco, social mobilization, agricultural modernization, modernity, regionalism, religion, know-how, territories, traditions, transmission of knowledge

\section{AUTEUR}

\section{SANDRINE SIMON}

Chercheuse, Centro de Estudos Interdisciplinares em Educação e Desenvolvimento (CeiED), Universidade Lusófona de Lisboa (Portugal)

sandrine.simon.dina@gmail.com 